\title{
A Laboratory-Centered Approach to Introducing Engineering Students to Electric Circuit and Electric Systems Concepts
}

\author{
Cyrus Shafai and Behzad Kordi \\ Department of Electrical and Computer Engineering \\ University of Manitoba, Winnipeg, Manitoba \\ Cyrus.Shafai@ad.umanitoba.ca, Behzad.Kordi@ad.umanitoba.ca
}

\begin{abstract}
The teaching of electric circuit analysis traditionally involves problem solving to ensure understanding of analysis theorems, complemented by laboratory experience. When taught to first year Engineering students, this approach lacks a motivational component and presents difficulties due to the weaker mathematics and problem solving skills of first year students. This paper presents a laboratory-centered approach to introduce engineering students to electric devices and systems. Using open-ended design projects, students explore and construct different types of electrical systems. Laboratories are selected so as to develop student intuition in electrical concepts, scientific fundamentals, provide a historical background, and demonstrate systems-level design issues. Over the past three years in our Department, using this approach, increased student motivation and engagement has been observed, supported by a significant increase in Electrical and Computer Engineering enrollment.
\end{abstract}

Keywords: Electric circuit, electronic device, laboratorycentered, discovery based learning, group learning.

\section{INTRODUCTION}

The education of engineering students in the subject of electric circuit analysis traditionally involves rigorous problem solving, complemented by laboratories that illustrate the various analysis theorems. This approach lacks a motivational component, which is more pronounced when the subject is taught to non-electrical or computer engineering students. Additional difficulties result when teaching first year engineering students, due to their weaker mathematics skills and immaturity in visualizing the abstract topology of circuit problems. For many students, a lack of intuition in electrical concepts further impedes understanding.

The difficulty in teaching the mathematical abstraction of circuit theory to early stage engineering students has been recognized by many. In [1], several factors were noted as causing difficulty in the teaching of electricity courses to engineering students from outside electrical and computer engineering. Some included lack of interest, the high level of abstract thinking required, the varied topics to be covered, and the use of complex number arithmetic. In addition, the teaching of electronics to students from other engineering fields is often done in service courses. Accordingly, large class sizes are common, and this was also noted in [1].

This paper presents a laboratory-centered approach to introduce engineering students to a broad range of electric devices and systems. This hands-on approach has been used for the past three years in our Department to teach first year, common core, engineering students electric concepts. Laboratory projects, many open-ended design projects, were selected so that students could explore and construct different types of electrical systems, including motors, communication systems, digital systems, medical instrumentation, and micro-electromechanical (MEMS) motion sensing systems. Many earlier studies have shown that an interactive approach to learning can benefit student retention and understanding [2,3]. Accordingly, it was felt that a laboratory-centered approach would be essential to achieve the broad topical content exposure. Our laboratory focus has been to provide a breadth approach, as opposed to a narrow analytical rigour based approach. Primary goals were to develop student intuition in electrical concepts and their scientific fundamentals, and to provide a historical background for the development of our modern electrical society.

This paper also discusses the pathway leading to the development of this teaching approach and teaching results achieved, which has included increased student motivation and a significant increase in Electrical and Computer Engineering enrollment.

\section{BACKGROUND MOTIVATION}

All $1^{\text {st }}$ year engineering students at the University of Manitoba undertake a common course program. In 
addition to courses in subjects such as Mathematics, Natural Sciences, English, and Computer Science, all students take three Engineering Science foundation courses: Introduction to Thermal Sciences, Introduction to Statics, and Introduction to Electrical and Computer Engineering.

The latter course introduces all engineering students to concepts in electrical systems. From 1999-2008, it focused primarily on electric circuit concepts using a traditional mathematics based approach. Experience has shown that while the ability to solve electric circuits problems is developed, this approach fails to give students an intuitive and contextual understanding of electrical systems. When teaching students who will subsequently continue in Electrical or Computer Engineering, this "circuit analysis first" approach is sufficient, as later courses provide more content and laboratory experience in various needed electronic device skills. However, students who later proceed into other fields, suffer from this lack of exposure to electrical systems content.

An additional issue is the mathematical skills level and problem solving maturity of $1^{\text {st }}$ year students. When circuit analysis is taught in $2^{\text {nd }}$ year, engineering students possess significantly stronger mathematical skills than those in $1^{\text {st }}$ year, who primarily rely on grade 12 level mathematics, and who commonly have never been exposed to Calculus or Complex Number concepts. In additional, $1^{\text {st }}$ year students have little experience in solving multi-step abstract problems. It is common that many circuit analysis problems provided in common textbooks are abstract, with multiple essentially arbitrary interconnections. They often have little relation with any physical electric circuits, and are rather focused on developing student mathematical skills in solving arbitrary circuit topologies.

It should be mentioned that the $1^{\text {st }}$ year courses in Thermal Sciences and Statics are also taught using a very analytical approach. However, in the case of these two courses, this approach works to strengthen a student's pre-existing understanding. Concepts such as heat conduction, temperature difference, liquid-gas phase, force loading and structure support are understood by students, at least at a low level. Such knowledge has already been gained through childhood experience, or has already been presented to students in science courses in earlier schooling. This background knowledge provides students some intuitive foundation, which helps them understand the physics behind the content taught in Thermodynamics and Statics courses. By comparison, this intuitive foundation is missing, for most students, in the subject of electrical systems, and so many students face the dual challenge of learning both electrical systems concepts and the mathematics of solving these systems under difference operational conditions.

Overall, two main educational motivations led to the revision of our $1^{\text {st }}$ year Introduction to Electrical and
Computer Engineering course. The first was to improve the ability of students to understand and solve basic circuit analysis problems involving both $\mathrm{dc}$ and ac sources, given their weak mathematics skills and lack of experience in solving abstract problems. Second, with electronic devices (such as sensors, actuators, microcontrollers) now ubiquitous, it is essential that future engineers, in all engineering disciplines, have an understanding of design principles and needs of a broad range of electrical devices.

\section{NEW COURSE STRUCTURE \& CONTENT}

\subsection{Course Structure}

Enabling the broad exposure to many electrical systems concepts, within the limited teaching time available, required a restructuring of the course delivery. It was decided that the traditional 3 lecture hours per week (39 hours/term) with 5 three hour laboratories (15 hours/term), totalling 54 contact hours, would not be suitable. With this limited number of laboratories, the desired additional electrical devices and systems content would need to be added to the lectures. This would be unsuitable, as lecture based teaching provides limited ability for students to gain practical experience.

Three solutions were considered. First, an initial restructuring was to restrict the circuit analysis teaching to only dc concepts, and to add additional laboratories, increasing from 5 to 8 while reducing their length to two hours. Feedback showed that while some additional electrical concepts could be introduced, the short laboratories only enabled simple circuits of narrow scope to be attempted. Also, restricting the course to only dc concepts, greatly limited the range of topics that could be introduced to students. As well, this limitation, to a degree, made the content that was delivered much less interesting leading a drop in student interest.

Second, consideration was to adopt a pure laboratory centered education. After consideration by faculty, this was rejected as it was strongly felt that students needed to have some degree of formal exposure to the methodology of solving electrical systems and circuit problems.

The third solution, which has now been implemented, was to structure the course with 2 lecture hours per week (26 hours/term) and 10 three hour laboratories, giving a course total of 56 contact hours. The lectures provide the formal exposure to solution methodologies, while the 10 laboratories provide the venue for practical exposure to working with and designing electrical systems.

Fall and winter teaching terms each have typically 200-220 students, divided between two lecture sections. Laboratories are given 5 days per week, resulting in 40-44 students in each laboratory. A smaller summer session also is taught. The laboratory room has 11 stations, resulting in groups of 3-4 students. This sub-division into 
smaller groups, with three TAs and a professor present in each laboratory session, solved the problem of the overall large class size. The group work also provides experience in collaborative learning. Students in laboratory groups submit individual reports (completed within each laboratory session). A combined group mark is also given based on the build quality of projects.

\subsection{Lecture Content}

The content of the lectures covers three main subjects. First, dc circuit analysis is covered in detail, using KVL and KCL methods, voltage and current division. Circuits are limited in complexity, as the goal is to teach and test the physics involved in the solution, as opposed to testing the ability of students to visualize and solve complex abstract circuit topologies. The advanced methods of Nodal or Loop analysis, and Source Transformations are not taught, and are left to $2^{\text {nd }}$ year courses. Typical circuits presented are limited to 2-3 loops.

Second, the solution of sinusoidal steady state ac circuits is covered. Concepts include phase, impedance, complex and average power, complex number mathematics (brief introduction), and capacitor and inductor devices. Solutions of circuits using impedance techniques are limited to 2 loops. The logic of limiting the circuit size is to not make circuits too large so that the solution becomes a demonstration of skill in complex number mathematics, as opposed to only demonstrating ability to solve steady state ac circuits. Complex number mathematics is covered more formally in other courses.

Third, digital logic concepts are taught. Topics include binary and hexadecimal numbers, number base conversion, 2's compliment mathematics, logic gates (AND, OR, NOT, etc.), truth tables \& functions.

Overall, the lecture content provides all $1^{\text {st }}$ year students a good formal exposure to the subject of dc and ac circuit analysis, and an introductory exposure to digital devices and logic. Students who later choose to enter electrical or computer engineering, would build upon this background in their later courses. This includes another formal circuit analysis course in their $2^{\text {nd }}$ year, which completes the subject, and which is undertaken at a more mathematically rigorous level.

\subsection{Laboratory Motivation \& Content}

The motivation for the 10 laboratories is to expose our common $1^{\text {st }}$ year students to advanced topics and electronic devices at an early stage. It is a benefit for all engineering students to possess a broad exposure to electrical systems. For example, mechanical engineers would benefit from some early experience with motors and digital systems. It is also a benefit for all engineering students to have experience with sensor systems, which are becoming ubiquitous, particularly inertial sensors.
The 10 three hour laboratories are structured as independent modules, where students are introduced to concepts surrounding the subject area of each laboratory. Each laboratory begins with a tutorial (typically 30-40 minutes) presented by a professor, that introduces the history, industrial implementation, and in some cases biological requirements, of the electrical systems to be utilized in the laboratory. Necessary background theory (scientific and circuit analysis methodology) is also presented. This is done so that each laboratory, to a degree, is independent of flow of the content delivery in the lectures. This was a requirement as students taking laboratories, on any of the 5 days per week, come from any of the various lecture sections of the course taught that term. Therefore, lecture content is not always synchronized between instructors and laboratories.

Each of the 10 laboratories is focused around a topical theme, such as electric motors, communication systems, biomedical devices, etc.. Many labs are open-ended projects, where student groups must construct a given electrical system (such as a self-leveling platform) out of basic electrical components and supplies (cardboard, tape, scissors, steel rod, popsicle sticks, etc.). This approach has three goals. First, to provide a Discovery Based Learning approach to topics and to build student intuition in electrical concepts and their scientific fundamentals. Second, by requiring students to actually build a physical device, they gain experience with practical thinking that all engineers need. Third, the lab projects are selected to provide an exposure to system level design concepts, such signal measurement, feedback, control, sensing, etc.. These issues are common to all engineering disciplines, and an exposure to them at the outset of their engineering education was thought to be beneficial.

The breadth of topics covered over the 10 laboratories is extensive. The first labs are introductory, providing students with no electrical experience some common background, and subsequent laboratories focus on more advanced topics. A brief summary of the 10 laboratories is given below. Pictures of some of the laboratory projects are given in Figures 1-4.

\section{Lab 1: Basic circuit concepts}

- Tutorial: Historical persona (Volta, Ampère, Ohm, Galvani, and others), voltage, current, series \& parallel resistors, power, the multimeter.

- Experiment: Build simple series and parallel resistor circuits, measure current, voltage, power.

Lab 2: KVL, KCL, incandescence, temperature effects

- Tutorial: Historical persona, incandescence and thermal emission, light spectrum, temperature effect on resistance, KVL, KCL, voltage and current division.

- Experiment: Build circuits using switches and light bulbs, measure resistance change with temperature. 
Lab 3: Power systems \& AC circuits

- Tutorial: Historical persona (Tesla, Westinghouse, Edison, Faraday, and others), ac \& dc power generation and transmission, RMS, frequency, Faraday's law of induction, oscilloscope, function generator.

- Experiments: Build transformer, build ac generator (using magnets, rod, cardboard, wire, tape).

Lab 4: Light sources, spectral efficiency

- Tutorial: Historical persona, arc lights, human vision, visible light efficiency, semiconductors, photoresistor, p-n junction, diode, LED, solar cell.

- Experiments: Diode circuits, build photoresistor light meter, compare efficiency of incandescent bulb to LED.

Lab 5: Motors \& transistors

- Tutorial: Historical persona, electromagnet, stepper \& dc motors, motor-generator duality, electric vehicles, BJT transistor as switch, optical position sensor.

- Experiments: Build single stator pole de motor using BJT optical rotation position sensor.

Lab 6: Amplifiers \& control systems

- Tutorial: Historical persona, ICs, cleanrooms, operational amplifiers, control systems (feedback, gain and stability), capacitors, tracking systems.

- Experiments: Build light tracking motor, DC motor as generator, build hand-crank flashlight with switched capacitor for energy storage.

Lab 7: Microsystems \& motion sensing

- Tutorial: MEMS, inertial sensors, hard drives, inkjet printing, touch screen technologies.

- Experiment: Build self-leveling platform using dc motor and MEMS sensor.

Lab 8: Biomedical technologies, EMG, ECG

- Tutorial: Historical persona, X-ray, MRI, ultrasound, and microwave tomography, the nervous system, the neuron, muscle function, EMG, heart function, ECG, measuring weak signals.

- Experiments: Build EMG and measure muscle signals vs. load, build ECG and measure heart signals at rest and after activity.

Lab 9: Communication and wireless technologies

- Tutorial: Historical persona, antennas, optical fibres, radar, phased array concept, electromagnetic waves, radio, AM \& FM modulation, FDMA-TDMA-CDMA cellular communication.

- Experiments: Build a capacitor for a RC low pass filter, build AM envelope detector with amplifier to demodulate and hear a music signal.

Lab 10: Digital systems

- Tutorial: Historical persona, analog and digital signals, sampling frequency, bit resolution (image and music examples), digital communication (coding, addressing, encryption concepts), binary numbers, digital logic gates, truth table, logic functions.

- Experiment: Logic circuits, build an optical burglar alarm with D-latch memory.

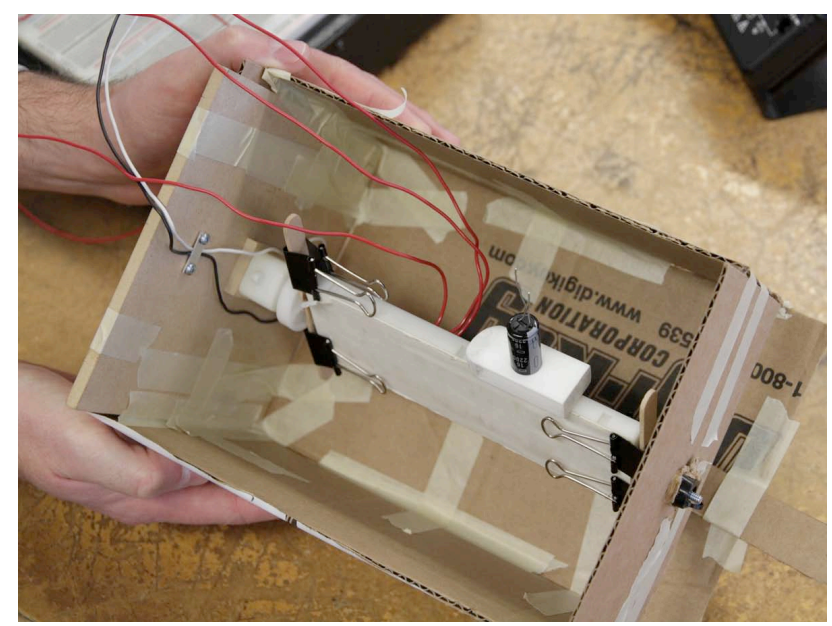

Figure 1: Student built self-leveling platform.

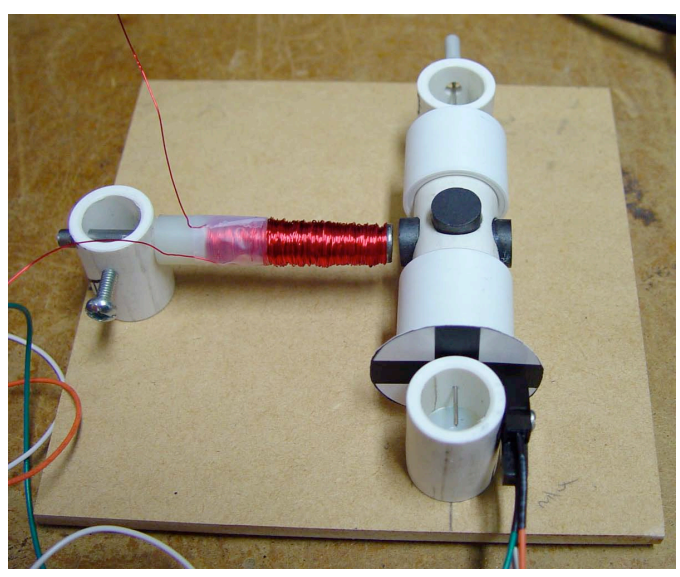

Figure 2: Feedback controlled dc motor.

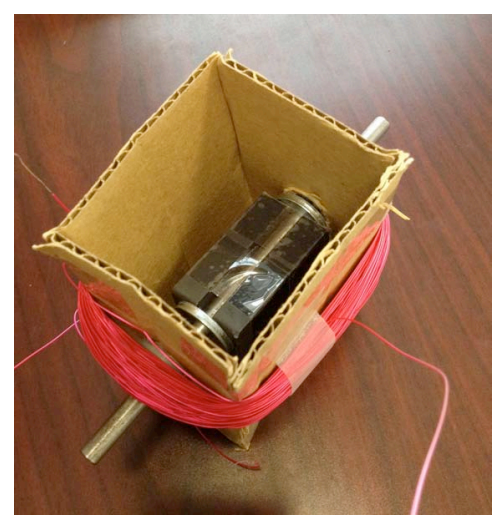

Figure 3: Simple ac generator. 


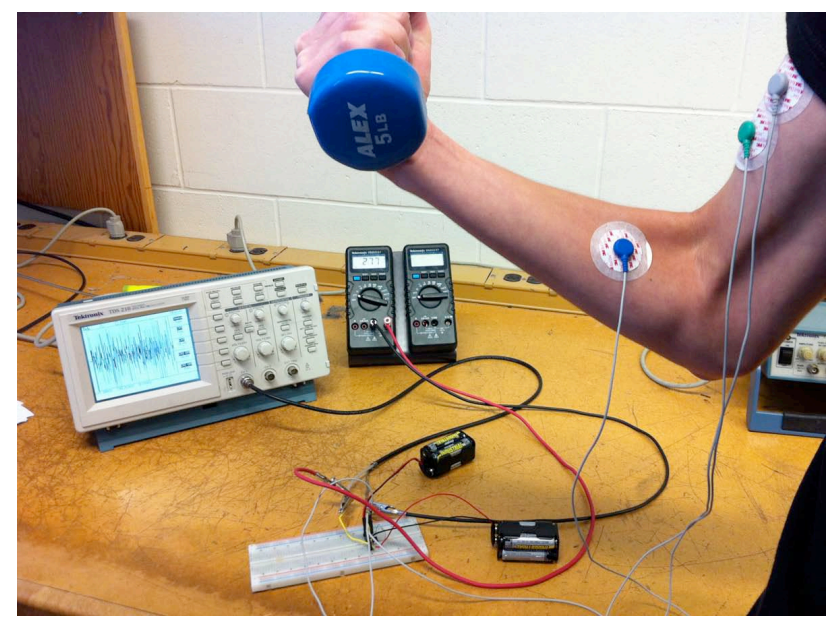

Figure 4: EMG measurement.

\section{RESULTS AND DISCUSSION}

The wide breadth of topics covered in the laboratories has impressed many of the faculty who have reviewed the content this course. The laboratory-centered approach has shown clear ability to introduce a very wide range of topics, with a hands-on experiential exposure, even to $1^{\text {st }}$ year students. This result supports the assertion that an interactive approach to learning can benefit retention and understanding.

The rational for this wide exposure was initially debated. For example, topics such as p-n junctions and transistors are only briefly introduced. Discussion as to whether these brief introductions, which lack the advanced mathematics and physics presentation done in upper year courses, would be worthwhile. Compromises were made, for example, the transistor is largely presented as a simple switch, with no formal discussion given for its usage as an amplifier.

Nonetheless, students have successfully shown the ability to construct, albeit simple, implementations of light meters, self-leveling tables, and light tracking motors. At the same time, they have gained experience using devices such as transistors and MEMS sensors.

Professors teaching this course have observed several facts. The most significant has been the increased student motivation observed in the laboratories, and significantly greater interest in the course subject than before. Also, greater student-professor and professor-student engagement is now occurring, with many students asking questions about advanced concepts beyond the scope of particular laboratories or lectures. Supporting these observations, is that enrollment into Electrical and Computer Engineering from the $1^{\text {st }}$ year into the $2^{\text {nd }}$ year has significantly increased. Enrollment since this course change has been implemented has increased 2.6 times.
Feedback from examinations has shown that students are capable of correctly solving circuit problems involving the wide range of topics presented in the new laboratories, such as transistor biasing, amplifier gain, and complex number impedance parameters for sinusoidal ac circuits (It should be noted that testing is now done using multiple choice examinations - where no parts marks are given, whereas in prior incarnations of the course part marks were given). This is in contrast to observations from the prior incarnation of the course, where large circuit analysis problems (often with arbitrary topology) were required of students. The prior lack of student ability to correctly solve these problems to full completion was often attributed to them to having poor understanding of the subject matter. Rather, it seems that in some cases the lack of ability was due to students not possessing the maturity to visualize and undertake multistep abstract problems, as opposed to them not understanding particular electric circuit or device concepts.

Essentially, students have shown that at the $1^{\text {st }}$ year level they are fully capable of understanding and applying the multitude of concepts covered the laboratories, as long as problem complexity does not extend beyond 2 or 3 levels. Extending beyond this level of complexity would require greater problem solving maturity than is commonly present at the $1^{\text {st }}$ year level. This being the case, perhaps a conclusion could be to leave some of the more complex problems for upper years when students have more maturity, and to instead spend the time teaching more background concepts, to develop a greater knowledge base before students enter $2^{\text {nd }}$ year.

The breadth of topics covered in the 10 labs, as well as the hands-on construction of the many small projects (such as those shown in Figures 1-3), gives students a broad conceptual exposure to design issues in many types electrical systems. This helps in the building of an intuitive understanding of concepts, which students can use in subsequent courses when they study these concepts in detail using formal mathematical analysis. It is hoped that this will help motivate students in more advanced upper year courses as well.

It should also be mentioned that the historical development and societal implications of technological innovations discussed in some of the laboratories could be of benefit to the education of engineering students. For example, the laboratory exploring energy efficient lighting begins with a discussion of lighting technology though history, from fire, to oil, to gas, then eventually to electric systems in various forms. This evolution at various stages has had significant societal and environmental consequences and benefits, such as indoor lifestyle, increased industrial productivity, literacy, and unexpected benefits such as inexpensive kerosene replacing whale oil (which can be attributed as one of the factors leading to the decline of the $19^{\text {th }}$ century whaling 
industry). These historical and societal discussions provide beginning engineering students with an awareness of how technological innovations have impacted society.

\section{CONCLUSIONS}

This paper presented our experience with teaching $1^{\text {st }}$ year common core engineering students in the subject of electrical devices and systems. The course was structured to have a greater laboratory contact time, than lecture time. Lecture content focused on formal exposure to circuit analysis mathematics. Laboratory time was focused on introducing engineering students to a broad range of electrical devices and systems concepts.

Developed laboratories introduced many concepts that are usually only discussed in upper year electrical and computer engineering courses. Students worked in groups to solve open-ended design projects within many of the laboratory sessions. Laboratory projects were selected to develop student intuition in electrical concepts and their scientific fundamentals, expose students to multiple electrical devices, and provide a historical background leading to the development of our modern electrical society. Also demonstrated in many projects are systems-level design issues common to all engineering disciplines, such as signal measurement, feedback and control, and sensing. Increased student motivation has been observed, supported by a significant increase in Electrical and Computer Engineering enrollment (an increase of 2.6 times), increased studentprofessor and professor-student engagement, with student inquiry about advanced concepts beyond the scope of the course now commonly occurring.

It should be noted that seven professors have taught sections of this course over the past three years, and similar results have been seen each year. This supports the conclusion that the outcomes are due to the course structure and content, as opposed to the person in front of the class.

\section{Acknowledgements}

The authors would like to acknowledge the other professors who have taught this course over the past 3 years. They include Drs. A. Gole, A. Major, J. Cai, S. Sherif, and D. Oliver.

\section{References}

[1] Khaled Arfa, Guy Oliver, and Georges-Émile April, "Basic Electricity Courses Revisited," in Proc. CEEA Canadian Engineering Education Conf., CEEC10, Andy Fisher (ed.) (Kingston, ON; 7-9 June 2010), 6 pp., 2010.

[2] M.J. Prince, "Does Active Learning Work? A Review of the Research," J. Engr. Education, The American Society of Engineering Education, 93(3), pp. 223-231, 2004.

[3] J. Imbert, H. Jahed, B. Owen, and G. Stubley, "Interactive Learning Applied in Undergraduate Mechancial and Mechatronics Courses at the University of Waterloo," in Proc. CEEA Canadian Engineering Education Conf., CEEC10, Andy Fisher (ed.) (Kingston, ON; 7-9 June 2010), 6 pp., 2010. 